\title{
Calculating Frequency of Occurrence of ASL handshapes
}

\author{
Jonathan Henner ${ }^{1}$ - Leah C. Geer ${ }^{2}$ - Diane Lillo-Martin ${ }^{3 *}$ \\ Boston University ${ }^{1} \cdot$ University of Texas at Austin $^{2} \cdot$ University of Connecticut $^{3}$ \\ jhenner@bu.edu·leah.geer@utexas.edu·diane.lillo-martin@uconn.edu
}

\begin{abstract}
Here we discuss an investigation of handshape markedness based on frequency of occurrence in an ASL database. Using a database of the most frequently used signs in a corpus of child language and other early-acquired signs we examined the handshapes of approximately 1000 signs by using two annotation systems, BTS and Stokoe annotation. Results indicate that the distribution of handshape use on the dominant and non-dominant hands is consistent with the predictions set forth by previous researchers in their examinations of handshape markedness. Our findings are also consistent with investigations handshape frequency in other sign languages, suggesting some cross-linguistic comparability in handshape markedness.
\end{abstract}

\section{INTRODUCTION}

$\mathrm{P}$ Honological markedness in the tradition of Bybee (1976), describes aspects of language production that are relatively difficult to perceive, produce, are acquired later by children, and occur less frequently within and across languages. In signed languages, discussions of markedness often focus on the handshape parameter. Here we discuss an investigation of handshape markedness in American Sign Language (ASL) based on frequency of occurrence in an ASL database.

Hierarchies of handshape markedness have been based on a variety of observations. BoyesBraem (1990) and Ann (1996) examined the anatomical and physiological properties of the hand and forearm to arrive at their respective predictions of markedness. Siedlecki \& Bonvillian (1997) noted the order in which children tend to acquire handshapes, and Lane et al. (1976) conducted an experiment in which revealed that unmarked handshapes are least often confused in visual perception tasks. Bat- tison (1978, among others) noted that these unmarked handshapes tend to be maximally distinct and occur in all sign languages studied to date (this includes very recent documentation on the phonology of hunting signs used by two groups in Botswana, Mohr \& Fehn, 2013). Finally, unmarked handshapes occur in a wide array of lexical contexts including use on the non-dominant hand.

While each of these researchers employed different methods to develop their respective predictions, they all arrived at roughly the same conclusion; there is a subset of handshapes used in ASL that are generally predicted to be less marked and should occur most frequently throughout the language. Figure 1 illustrates the seven handshapes Battison predicts to be maximally unmarked.

In the present investigation, what is at issue is that, while Battison proposed seven distinct unmarked handshapes, others have proposed as few as three or four (Sandler, 1996). New frequency statistics can provide relevant evidence for this discussion.

\footnotetext{
* The project described here is supported by Award Number R01DC009263 from the National Institute on Deafness and other Communication Disorders of the National Institutes of Health. The content is solely the responsibility of the authors and does not necessarily represent the official views of the NIDCD or the NIH. We would also like to thank attendees of the LSA 2013 Annual Meeting who provided us with insightful comments about the present work and ways in which we can improve and expand up on it in the future.
} 


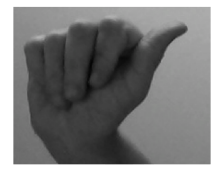

A

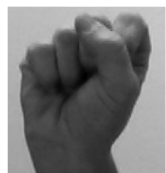

S

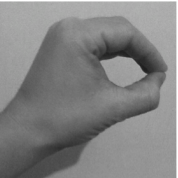

$\mathrm{O}$

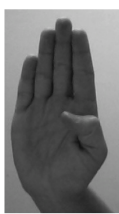

B

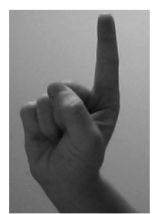

$1 / \mathrm{G}$

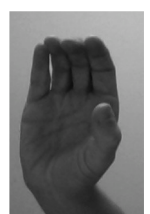

C

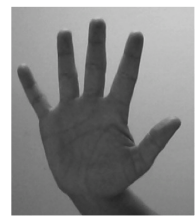

5

Figure 1: Subset of maximally unmarked handshapes, according to Battison

\section{Methods}

Using an ASL ID Gloss database (see Alkoby et al., 2010; Fanghella et al., 2012, for discussion of creation and recent implementation), we examined the handshape parameter in the dominant and non-dominant hands of approximately 1000 signs produced in citation form by several adult signers. Roughly half of these signs were extracted and reproduced from spontaneous signing in a corpus of child and child-directed language (Chen Pichler et al., 2010). The remaining signs were elicited translations of lexical items from Fry's Word List (Fry, 2004), a collection of high frequency words used for reading and writing. A strength to our approach was using multiple handshape notation systems. All signs were coded using Stokoe Notation (Stokoe et al., 1965) and the Berkeley Transcription System (BTS) (Hoiting \& Slobin, 2002; Kuntze, 2007). Stokoe et al.'s notation system glosses over some possibly noncontrastive differences (e.g., handshapes A, S, T are all assigned the same designation), whereas BTS captures more precise distinctions in hand configuration, giving separate designations to varieties of related handshapes.

\section{Results}

The most frequent handshapes in our database were $\mathrm{B}, 5, \mathrm{~A}, \mathrm{G} / 1$ which is consistent with predicted frequency and handshape acquisition order previously noted from ASL and other sign languages including British SL (Mann et al., 2010), Brazilian SL (Karnopp, 2002), and Taiwanese SL (Ann, 2005). One interesting finding is the inclusion of the B handshape, contrary to Sandler's (1996) analysis of B as more marked. The relatively lower frequency of $\mathrm{C}$ is consistent with her analysis, which excludes it from the unmarked set. With our two annotation systems, we can see that while BTS shows more detail, it still captures the same generalizations as Stokoe notation. Figures 2a and 2a illustrate the distribution of handshapes with each notation system. Also, handshapes used on the non-dominant hand were almost exclusively from the unmarked set (B, 5, A, G/1) in our data confirming Battison's prediction that the weak hand should bear unmarked handshapes. Figure 3 demonstrates the distribution of non-dominant handshapes; $B$ handshapes occur with roughly equal frequency regardless of which notation system is used, further suggesting their unmarked status.

\section{Discussion \& Future WORK}

Results indicate that the distribution of handshapes in this database is consistent with the predictions set forth by previous researchers but the set of maximally unmarked handshapes is only a subset of those pictured in Figure 1. With this evidence, it certainly seems to be the case that ASL favors unmarked handshapes. Furthermore, this set of handshapes corresponds to child handshape acquisition and the most frequently occurring handshapes from other languages including British SL (Mann et al., 2010), Brazilian SL (Karnopp, 2002), and Taiwanese SL (Ann, 2005). Additionally, some of these handshapes are used in the secondary sign systems used for hunting by certain tribes in Botswana (Mohr \& Fehn, 2013). This suggests fairly robust cross-linguistic comparability in handshape markedness. 


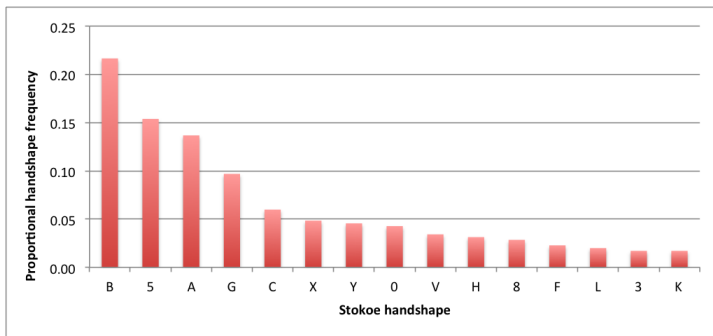

(a) Stokoe Hs Distribution

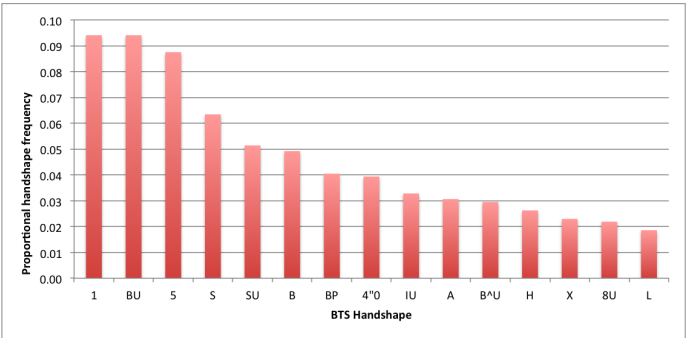

(b) BTS Hs Distribution

Figure 2: Hs distributions using two different notation systems

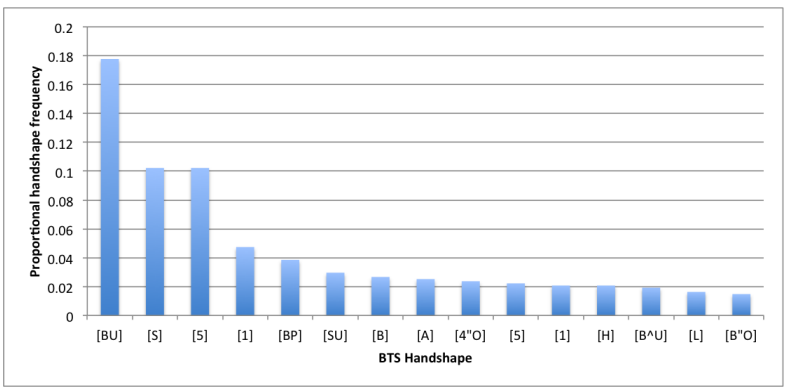

Figure 3: Non-dominant Hs Distribution (BTS Notation)

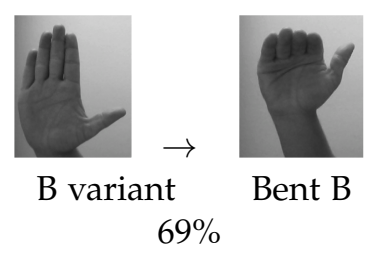

(a) BTS notation

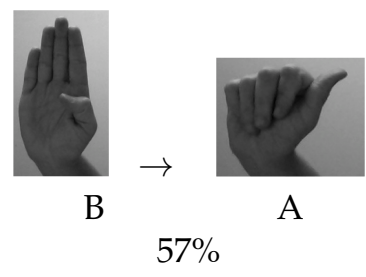

(b) Stokoe notation

Figure 4: Frequent Hs bigrams: This figure indicates that, for (a), $69 \%$ of the time the B variant handshape begins a sign, a bent B results from a handshape change. For (b), 57\% of the time a B handshape begins a sign, a handshape change will result in an A handshape.

There are two ways in which we hope to further this project and continue to make use of this database. First, we would like to reexamine the extent to which the Symmetry and Dominance conditions Battison (1978) suggested are borne out in this database. We showed that handshapes used on the nondominant hand are, for the most part, conform to those unmarked forms per Battison's stip- ulation, however other handshapes were also used. How often and in what particular signs are more marked handshapes used on the nondominant hand? Does this pose a problem for assumptions we have made about the phonological grammar?

Next, we would like to examine handshape bigram frequencies. Specifically, given handshape $X$, how likely is handshape $Y$ ? This 
would prove useful in controlling for frequency of handshape and handshape transitions in psycholinguistic experiments.

Earlier we noted that while Sandler suggested that the set of unmarked handshapes did not include B, we found this to be a prevalent handshape in our database. Related to this discussion and with respect to handshape bigrams consider the findings from our database presented in Figure 4. Of note is that of signs with handshape changes, $\mathrm{B}$ and related handshapes are still quite prevalent - regardless of notation system, B and related handshapes occur frequently.

\section{REFERENCES}

Alkoby, K., Hochgesang, J., Mirus, G., \& Pascual Villanueva, P. (2010). Construction of an ID Gloss database. In Proceedings from the $10^{\text {th }}$ meeting of the Theoretical Issues in Sign Language Research Conference, West Lafayette, IN.

Ann, J. (1996). On the relation between ease of articulation and frequency of occurrence of handshapes in two sign languages. Lingua, 98:19-41.

Ann, J. (2005). A functional explanation of Taiwan Sign Language handshape frequency. Language and Linguistics, 6(2):217-246.

Battison, R. (1978). Lexical borrowing in American Sign Language. Linstok Press, Silver Spring.

Boyes-Braem, P. (1990). Acquisition of handshape in American Sign Language: A preliminary analysis. In Volterra, V., editor, From Gesture to Language in Hearing and Deaf Children, pages 107-127. Springer, New York.

Bybee, J. (1976). An Introduction to Natural Generative Phonology. Academic Press, New York.

Chen Pichler, D., Hochgesang, J. A., Lillo-Martin, D., \& Müller de Quadros, R. (2010). Conventions for sign and speech transcription of child bimodal bilingual corpora in ELAN. Language, Interaction and Acquisition/Langage, Interaction et Acquisition, 1(1):11-40.

Fanghella, J., Geer, L., Henner, J., Hochgesang, J., Lillo-Martin, D., Mathur, G., Mirus, G., \& Pascual
Villanueva, P. (2012). Linking an ID-gloss database of ASL with child language corpora. In Proceedings of the $5^{\text {th }}$ Workshop on the Representation and Processing of Sign Languages: Interactions between Corpus and Lexicon at Language Resources and Evaluation (LREC), pages 57-62, Istanbul, Turkey.

Fry, E. (2004). 1000 instant words: The most common words for teaching reading, writing, and spelling. Teacher Created Resources, Westminster, CA.

Hoiting, N. \& Slobin, D. (2002). Transcription as a tool for understanding: The berkeley transcription system for sign language research BTs. In Morgan, G. \& Woll, B., editors, Directions in Sign Language Acquisition. John Benjamins, Amsterdam.

Karnopp, B. (2002). Sign phonology acquisition in Brazilian Sign Language. In Morgan, G. \& Woll, B., editors, Directions in Sign Language Acquisition. John Benjamins, Amsterdam.

Kuntze, M. (2007). Hand Configuration Notation Guidelines for BTs. Boston University, Boston, MA.

Lane, H., Boyes-Braem, P., \& Bellugi, U. (1976). Preliminaries to a distinctive feature analysis of handshapes in American Sign Language. Cognitive Psychology, 8(2):263-289.

Mann, W., Marshall, C. R., \& Morgan, G. (2010). The acquisition of sign language: The impact of phonetic complexity on phonology. Language Learning and Development, 6(1):60-86.

Mohr, S. \& Fehn, A. M. (2013). Phonology of hunting signs in two kalahari khoe-speaking groups (ts'ixa and I I ani). In Handbook of the $87^{\text {th }}$ Annual Meeting of the Linguistics Society of America, pages 183-184, Boston, MA.

Sandler, W. (1996). Representing handshapes. International Review of Sign Linguistics, 1:115-158.

Siedlecki, T. \& Bonvillian, J. D. (1997). Young children's acquisition of the handshape aspect of American Sign Language signs: Parental report findings. Applied Psycholingustics, 18:17-39.

Stokoe, W. C., Casterline, D. C., \& Croneberg, C. G. (1965). A dictionary of American Sign Language on linguistic principles. Linstok Press, Silver Spring, MD. 